Voix et Images

voixetimages

\title{
Mourir. En attendant, dire « Bof »...
}

\section{Pierre Hébert}

Volume 13, numéro 2 (38), hiver 1988

Le propre du corps Roger Des Roches

URI : https://id.erudit.org/iderudit/200718ar

DOI : https://doi.org/10.7202/200718ar

Aller au sommaire du numéro

\section{Éditeur(s)}

Université du Québec à Montréal

\section{ISSN}

0318-9201 (imprimé)

1705-933X (numérique)

Découvrir la revue

\section{Citer cet article}

Hébert, P. (1988). Mourir. En attendant, dire « Bof »... Voix et Images, 13(2),

339-343. https://doi.org/10.7202/200718ar d'utilisation que vous pouvez consulter en ligne.

https://apropos.erudit.org/fr/usagers/politique-dutilisation/ 


\section{Roman}

\section{Mourir. En attendant, dire «Bof»...}

\section{par Pierre Hébert, Université de Toronto}

Les politiciens parlent de politique, les écrivains, de littérature, les gens heureux, du bonheur, les gens pauvres, parfois de la pauvreté, et c'est ainsi que les paroles coulent, que les livres s'écrivent. Mais qui parle de la mort? Les vivants, bien sûr, et c'est alors que les choses risquent de se gâter. Car à parler de la mort, le langage se trouve en quelque sorte acculé à ses limites: signifier un inconnu non de degré, mais de nature. À moins que la mort soit le nec plus ultra des sujets littéraires, celui qui exige la plus intense des créations par cette projection dans un Autre totalement inconnu? Quoi qu'il en soit, voilà bien un sujet que les lettres québécoises n'hésitent pas à fréquenter: Agonie (Jacques Brault), Lucie ou un midi en novembre et les Heures (Fernand Ouellette), la Convention (Suzanne Lamy) et, ici quatre textes de prose où la mort se profile. Quant au cinquième... «bof». 


\section{Certains se déplacent, d'autres voyagent}

Acceptera-t-on que cette chronique s'ouvre avec un texte qui est paru à la fin de 1986, mais dont on a assez peu fait état? Rien que de banal, à première vue, à la fois dans le titre et le genre de ce Voyage d'hiver ${ }^{1}$ de Jean Éthier-Blais. Pris prosaïquement, ce texte raconte un voyage fait dans une saison définie; le texte sera donc simple, comme tous ces récits de voyage où s'amalgament le carnet, le journal, dans un recueil de souvenirs et d'impressions qui risquent de n'intéresser que celui qui a déjà fréquenté les mêmes chemins que l'auteur, en l'occurence l'Italie, Paris, Genève, Zurich... Mais attention: un voyage en cache peut-être un autre.

Dans son Journal philosophique et littéraire, François Hertel écrivait que l'égoïsme était de rigueur: on peut en dire autant dans le cas de ce récit de Jean Éthier-Blais. Car cet égoïsme sain consiste à imprégner la diversité des espaces que l'on parcourt, d'une force centripète qui n'est rien d'autre que soimême. Il s'agit non pas d'aller vers du nouveau, mais, par d'imperceptibles gradations, de se découvrir, une fois de plus, tel qu'en soi-même (p. 9). La multiplicité des lieux que parcourt Jean Éthier-Blais dans son récit ne doit pas faire écran: l'on revient toujours à soi. C'est ainsi que lorsque le voyageur est rempli d'émoi devant un tableau, lorsqu'il éprouve une nostalgie immense devant Venise et ses canaux, c'est de lui-même dont il est question, c'est-à-dire de nous tous. L'objet du parcours renvoie au sujet qui nous est intimement lié dans l'humanité que nous partageons avec lui. Et ce que nous avons le plus en commun, c'est de faire ce même voyage d'hiver en direction de la mort.

Car la mort est omniprésente dans ce beau texte. Dès le premier chapitre, le récit de voyage est interrompu par la mort de René Garneau et par des réminiscences à son sujet. Que cherche ce voyageur? Je n'en sais rien, sinon que je suis porté par un désir insatiable, moi homme d'Amérique, comme ces voyageurs du Nord qui veulent trouver ici, à l'instar de Goethe, la réalisation suprême de soi; ou comme Stravinski, une tombe (p. 62). Il s'établit chez le voyageur une filiation entre ce qu'il voit et lui-même, comme par exemple devant. Venise: On est à Venise devant le cheminement en soi de la décrépitude, de la maladie, de la mort. [...] La vie est semblable à cette ville belle entre toutes. Elle est minée (p. 79]. En vérité, plus il avance dans la lecture de ce Voyage d'hiver, plus le lecteur comprend que le parcours des lieux, que le récit même avance vers la mort: Ce récit de voyage, qui est aussi l'occasion de souvenirs, est une suite de digressions, de remarques a propos d'autre chose. [...] À l'intérieur de ce récit, j'ignore où me porteront mes pas, sinon d un tombeau (p. 157). Jean Éthier-Blais ne se risque cependant pas au-delà de la simple expérience qui consiste à savoir que l'on va mourir, mis à part quelques éclairs vifs: Mort, transfiguration de la vie. Existences gigognes (p. 87). Certes, ce Voyage d'hiver est superbement écrit, et il s'y manifeste une culture exceptionnelle; mais j'ai été frappé avant tout par la vision que ce voyageur portait, en lui, de sa propre mort (p. 99). 


\section{Qui a prétendu que les morts étaient morts?}

Quand on ouvre un récit de Daniel Gagnon, on peut être certain que le texte, dans sa brièveté, s'affirmera néanmoins par sa densité et par un accent à la fois de tourment et d'urgence. Je pense ici en particulier à la Fille à marier, une série de lettres d'une toute jeune adolescente à une interlocutrice imaginaire à Medicine Hat. Feignant de parler à l'autre, la narratrice se racontait à elle-même son propre désespoir.

Dans la Fée calcinée2, Daniel Gagnon ne procède pas autrement. La narratrice est à nouveau une femme, et son discours épouse les apparences d'une longue lettre, ou plutôt d'un long chant désespéré adressé à divers destinataires. La gravité de cette complainte nous saisit dès le début: car c'est de sa tombe que la narratrice parle.

L'impossible dialogue entre les vivants et les morts se trouve ici renversé: c'est la morte qui s'adresse aux vivants qui ne l'entendent pas. Imaginez plutôt: la morte est dans son cercueil, mais elle n'est pas morte, car la Mort n'a pas encore daigné venir la prendre. La narratrice erre entre deux mondes: Mourante mais incapable de mourir, épuisée de fatigue, je me couche, je me précipite la tête dans mon cercueil et j'attends, j'appelle la mort (p. 18). Touchée par la Mort mais non point morte, dit-elle elle-même, elle regarde les vivants s'agiter et, à la manière des matelots de l'Oceano nox de Victor Hugo, elle se voit mourir dans la mémoire des vivants: $\hat{O}$ priez pour moi jeunes gens! ne m'oubliez pas, n'oubliez pas vos morts, si seuls et si perdus, errant dans le néant et se morfondant, priez pour qu'ils trouvent enfin la paix! (p. 24). La narratrice verra son souhait exaucé: la Mort viendra en effet la chercher.

Cliché de salon mortuaire: la mort est plus difficile à supporter pour ceux qui restent que pour ceux qui ont trépassé. Qu'en sait-on au juste? Ce récit de Daniel Gagnon s'immisce au cœur d'une morte qui hurle au dedans d'elle-même sans fin, d'une morte plus triste et plus seule encore que ne peuvent l'être les vivants pour qui, au moins, la vie continue.

\section{La marche à la mort}

Marcher est une nécessité vitale. J'actionne le réel, je fais défiler les multiples, j'accélère la modification des apparences. Quand je marche, je me projette dans un couloir d'événements (p. 14). Ce que Paul Chamberland propose, dans Marcher dans Outremont ou ailleurs ${ }^{3}$, c'est un voyage dans le couloir de la vie et des embûches qui le jalonnent. Marcher, vivre quoi, c'est s'exposer à l'autre, à son incompréhension, à sa morale qui étouffe le désir: Les singeries de la morale ne mènent qu'à la stérilité spirituelle et s'accomodent fort bien du matérialisme comptable, de l'animalité la plus morte, minéralisée (p. 51). Qui sème des oiseaux morts sur mon chemin?, s'écrie le marcheur (p. 46). Et la marche dans la vie est en même temps une marche à la mort: J'irai. Je voudrai l'engloutissement vivant dans le trou de moi (p. 27 ). 
Mais de quel genre de récit s'agit-il? Marcher dans Outremont ou ailleurs tient du journal en ce qu'il est une notation d'impressions, un ghetto des âmes (p. 66). Mais ce serait illusion de croire que le marcheur est seul: Paul Chamberland parsème son texte de citations en regard, et sa parole devient solidaire de l'autre, du moins de celui qui rejoint ses propres préoccupations auxquelles renvoie l'écho intertextuel. Et lire ce récit, c'est accepter une marche analogue à celle de la vie: sans savoir vers quel détour la parole nous entraîne, sauf la mort. En attendant, une grande souffrance, car refuser la souffrance, c'est inévitablement l'entretenir (p. 75).

\section{Une histoire de serpents}

Du Voyage d'hiver de Jean Éthier-Blais à l'Hiver au cœur ${ }^{4}$ d'André $^{\prime}$ Major, rien de commun à première vue, hors le titre. Cette nouvelle d'André Major raconte l'histoire d'Antoine et des problèmes courants vers trente ans, quand tout ou à peu près se mit à aller de travers (p. 11). Avec un art condensé où subtilement un certain sourire de l'esprit tempère la gravité de la situation, André Major exprime bien le désir d'Antoine de vouloir tout abandonner, de chercher à disparaître de la carte des humains jusqu'à ce que, ainsi livré à luimême, il renoue avec son enfance. Car, démuni de tout, Antoine redevient curieusement attentif à tout: aux odeurs, aux sons, aux états d'âme. La quête d'Antoine est celle d'un salut personnel sans prétention, mais vrai, comme tout le récit d'ailleurs: C'est ainsi qu'en tentant de rompre avec son passé, il était revenu sur ses pas, dans la promiscuité des origines, pour tomber amoureux de quelqu'un qui parlait avec l'accent de son enfance (p. 57).

Mais au début du récit, il y avait la mort, ou plutôt ce désir de mort: Antoine se prit à souhaiter très fort de disparaître dans le paysage qui s'étalait, blanc et fade [...] (p. 14). Amorcé à l'ombre de l'hiver et de la mort de l'âme, le récit touchant d'Antoine reprend la direction de la vie. Le serpent humain, comme en témoigne l'exergue de Pavese, ne peut se défaire de sa vie passée; la tentation du nihilisme s'efface devant la redécouverte d'un plaisir innocent de vivre, devant la rencontre d'une amie de jadis, Huguette:

Ce n'était plus l'hiver qu'il avait au cour - un hiver qu'il avait cru impérissable -, c'était l'image brûlante d'Huguette, son rire clair, l'extrême douceur de sa peau et cette apparente légèreté qu'elle apportait à tout ce qu'elle touchait mais que démentait parfois son regard de naufragée (p. 46).

La rapidité de l'esquisse d'une situation, la justesse du ton, certes, mais peutêtre avant tout l'art naturel, simple et juste plaira dans ce court texte; au surplus, la recherche de soi du personnage n'a rien d'isolé et devrait émouvoir tout lecteur qui n'a pas encore minéralisé sa vie, pour reprendre le mot de Paul Chamberland. 


\section{Et en attendant, dire $\ll$ Bof $* .$.}

Bon, d'accord, les romans mystiques sont populaires par les temps qui courent: Agonie, la Constellation du Cygne, Lucie ou un midi en novembre, textes d'élévation, si l'on peut dire, où les personnages vont au Tibet, à Assise ou... en plein Cosmos. Mais n'oublions pas les «romans-bof»: la Note de passage, Encore une partie pour Berri, Loft Story et, ici, le bof des bofs, Bof génération 5 de Jean-Yves Dupuis. Le héros-type de ce genre de roman est bien sûr désabusé, cynique, délibérément sans idées. Son ennemi numéro un: l'idéologie, qui n'existe que pour servir celui qui prétend s'en servir. Fils ou filles d'Hervé Jodoin? Pas tout à fait, car une nouvelle caractéristique les distingue: ces personnages sont jeunes, la plupart du temps.

Dans Bof génération, le désabusement semble à première vue parvenir à un extrême. Le personnage, du même nom que l'auteur, se méfie de tout ce qui bouge et qui s'appellerait idée: les idées ne valent pas la peine d'être débattues (p. 12), n'hésite-t-il pas à dire, et ses discussions préférées sont celles dont le contenu est parfaitement insipide, sans prétention de refaire le monde. Arrive inopinément une fille, Nadine, qui s'installe chez Jean-Yves sans avoir vraiment été invitée. Alors survient un petit drame sans trop d'importance (Nadine tue quelqu'un pour voler) qui dérange quelque peu leur vie, et qui leur donne même le goût de parler. Mais n'exagérons rien: à la fin, Nietzche est mort. Dieu aussi (p. 135).

Ceci n'était pas un résumé, bien sûr. Comment se prêter à pareil exercice, quand l'auteur clame: mon roman raconte une histoire débile... Alors donnons dans le débile à plein, c'est-à-dire dans le faible, dans ce qui n'a pas de consistance: les grandes idées, les causes exaltantes agonisent, «avoir raison» est possessif et suspect car la raison universelle n'existe plus. Néanmoins, il faudrait peut-être éviter, dans un jugement rapide, de croire que ces personnages sont entièrement désabusés, qu'il ne leur reste qu'à vivoter en attendant la mort. Leur acte de foi est peut-être fondé sur la valeur de la personne, accompagnée d'une immense tolérance. Voilà comme je suis: je me mêle de faire le procès de la société, et je ne suis pas fichu de faire une compote de pommes. Quel imbécile je fais! (p. 52).

Il faut tout de même avoir des priorités dans la vie.

1 Jean Éthier-Blais, Voyage d'hiver, Montréal, Leméac, 1986, 173 p.

2 Daniel Gagnon, la Fée calcinée, Montréal, VLB/Le Castor astral, 1987, $115 \mathrm{p}$.

3 Paul Chamberland, Marcher dans Outremont ou alleurs, Montréal, VLB, 1987, $106 \mathrm{p}$.

4 André Major, l'Hiver au cœur, Montréal, XYZ éditeur, 1987, 77 p.

5 Jean-Yves Dupuis, Bof génération, Montréal, Québec/Amérique, 1987, $140 \mathrm{p}$. 